University of Nebraska - Lincoln

DigitalCommons@University of Nebraska - Lincoln

October 2013

\title{
Prevalence, Serotype, Virulence Characteristics, Clonality, and Antibiotic Susceptibility of Pathogenic Yersinia enterocolitica from Swine Feces
}

\author{
Saumya Bhaduri \\ USDA-ARS, saumya.bhaduri@ars.usda.gov \\ Irene V. Wesley \\ National Animal Disease Center
}

Follow this and additional works at: https://digitalcommons.unl.edu/usdaarsfacpub

Bhaduri, Saumya and Wesley, Irene V., "Prevalence, Serotype, Virulence Characteristics, Clonality, and Antibiotic Susceptibility of Pathogenic Yersinia enterocolitica from Swine Feces" (2013). Publications from USDA-ARS / UNL Faculty. 1233.

https://digitalcommons.unl.edu/usdaarsfacpub/1233

This Article is brought to you for free and open access by the U.S. Department of Agriculture: Agricultural Research Service, Lincoln, Nebraska at DigitalCommons@University of Nebraska - Lincoln. It has been accepted for inclusion in Publications from USDA-ARS / UNL Faculty by an authorized administrator of DigitalCommons@University of Nebraska - Lincoln. 


\title{
Prevalence, Serotype, Virulence Characteristics, Clonality, and Antibiotic Susceptibility of Pathogenic Yersinia enterocolitica from Swine Feces
}

\author{
Saumya Bhaduri and Irene V. Wesley
}

\subsection{Introduction}

Enteroinvasive pathogenic strains of Yersinia enterocolitica are recognized as major human pathogens that cause 96,000 cases of human disease annually in the United States (Scallen et al. 2011). Ninety percent of those cases are the result of foodborne transmission (Centers for Disease Control and Prevention (CDC) 2006; Nesbakken 2005; Scallen et al. 2011). Swine are identified as important reservoirs of $Y$. enterocolitica serotypes (O:3, O:5, O:8, O:9) that are associated with human illness (Fredriksson-Ahomaa et al. 2011). Pathogenic $Y$. enterocolitica carriage in swine ranges from 35 to $70 \%$ of herds and 4.5 to $100 \%$ of individual pigs (Ortiz Martinez et al. 2009). $Y$. enterocolitica is transmitted among swine by the oral-fecal route and is found on the surface of freshly slaughtered pig carcasses. This is likely the result of the spread of feces or contamination from the oral cavity during the slaughtering process (Laukkanen et al. 2009). In the

\footnotetext{
S. Bhaduri $(\bowtie)$

Molecular Characterization of Foodborne Pathogens Research Unit, Eastern Regional Research Center, USDA/NAA/ARS, 600 E. Mermaid Lane, Wyndmoor, PA 19038, USA

e-mail: saumya.bhaduri@ars.usda.gov

I.V. Wesley

Food Safety and Enteric Diseases Research Unit, National Animal Disease Center, Wyndmoor, PA, USA
}

United States, few studies on the prevalence of $Y$. enterocolitica in swine have been conducted (Bhaduri 2001; Bhaduri and Wesley 2006; CDC 2006, 2011).

Since pork safety begins on the farm, producers and practitioners play a critical role in providing safe products. Therefore, an investigation was conducted as a part of the U.S. Department of Agriculture (USDA) National Animal Health Monitoring System (NAHMS) Swine 2000 Study to measure the distribution of pathogenic Y. enterocolitica in finisher pigs and thus to identify potential control factors to reduce the public health risk factors associated with this pathogen. This study represented $92 \%$ of the U.S. hog inventory and $75 \%$ of its operations from the top 15 hog-producing states. Since $Y$. enterocolitica is a commensal organism of swine, fecal samples were tested for its occurrence. Regulatory policy states that isolation of a pathogen is an essential requirement in pathogen monitoring programs. Thus, enrichment, isolation, and verification by a fluorogenic PCR assay were used to monitor the prevalence of pathogenic $Y$. enterocolitica. The pathogenic potential of the individual isolates was assessed by serotyping, screening for the presence of the plasmid for Yersinia virulence (pYV), and testing for virulence-associated determinants, which were then correlated with genotype, expression of YopE protein, and antibiotic susceptibility. The information generated will be useful for the identification of on-farm management and processing practices leading 
to $Y$. enterocolitica contamination. Modification of such practices would ultimately result in reducing $Y$. enterocolitica transmission from pork products to humans.

\subsection{Materials and Methods}

\subsubsection{Study Design and Sample Collection}

On-farm sampling collections were conducted by USDA-Animal and Plant Health Inspection Service (APHIS) federal and state field veterinarians from September 6 through December 6, 2000 and January 3 through March 20, 2001 as previously described (Bhaduri and Wesley 2006; Bhaduri et al. 2005, 2009).

\subsubsection{Isolation of Pathogenic Yersinia enterocolitica}

The overall process for isolation and characterization of presumptive $Y$. enterocolitica isolates is depicted in Fig. 14.1. Identification of $\mathrm{CIN}^{+}$presumptive clones as ail positive pathogenic Y. enterocolitica by fluorogenic 5' nuclease PCR assay targeting the chromosomal virulence ail gene (attachment invasion locus); storage of ail $^{+}$ $Y$. enterocolitica isolates; pYV screening in ail-positive $Y$. enterocolitica; determination of pYV-associated virulence determinants; detection of YopE in cell lysates by sodium dodecyl phosphate-polyacrylamide gel electrophoresis (SDSPAGE); and Western blotting were carried out as previously described (Bhaduri and Wesley 2006; Bhaduri et al. 2005).
Fig. 14.1 Flow chart for isolation and characterization of presumptive Yersinia enterocolitica isolates
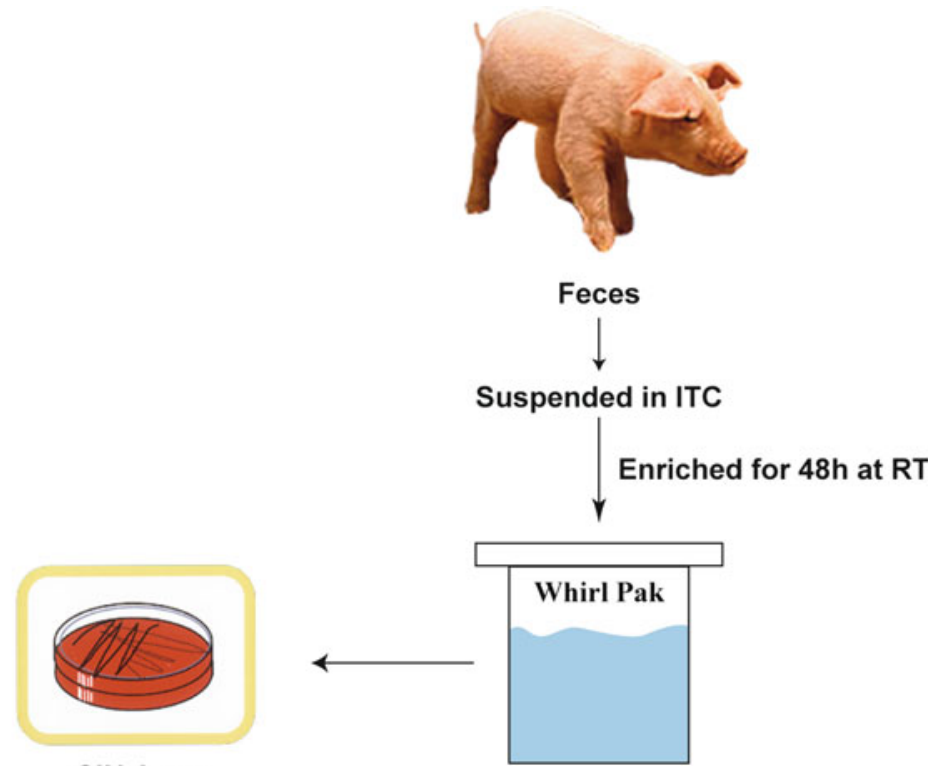

CIN Agar

Incubate for $48 \mathrm{~h}$ at $25^{\circ} \mathrm{C}$

Isolate and Confirm with PCR

targeting ail gene 


\subsubsection{Assessment of the Pathogenic Potential of the Isolates}

The ail-positive $Y$. enterocolitica isolates were serotyped as previously described (Bhaduri and Wesley 2006); Pulsed-Field Gel Electrophoresis (PFGE) and cluster analysis were performed as described (Bhaduri et al. 2009) and antimicrobial susceptibility testing was determined as described (Bhaduri et al. 2009).

\subsection{Results and Discussion}

\subsubsection{Prevalence}

A total of 2,793 swine fecal samples from 77 production sites were surveyed for the presence of pathogenic $Y$. enterocolitica over a period of
27 weeks (September 6, 2000 to December 6, 2001 and January 3 through March 20, 2001). The farms were located in 2 eastern and 13 mid-western states. Figure 14.2 shows the states that participated in the swine study. A fluorogenic $5^{\prime}$ nuclease PCR assay (Bhaduri et al. 2005) detected the chromosomal ail gene in 345 of 2,793 enrichments. Samples were used for the presumptive isolation of $Y$. enterocolitica on selective CIN agar, and $\mathrm{CIN}^{+}$presumptive colonies were then identified as pathogenic $Y$. enterocolitica by a fluorogenic $5^{\prime}$ nuclease PCR assay targeting the chromosomal ail gene. A total of 106 (3.8\%) $\mathrm{CIN}^{+}$(ail-positive) Y. enterocolitica colonies were isolated from 2,793 total fecal samples and evaluated for pathogenic potential. In this study, prevalence based on fecal samples was higher $(3.8 \%)$ than the recent $0.5-1.4 \%$ estimate for pigs surveyed in Europe (FredrikssonAhomaa et al. 2011; Laukkanen et al. 2009;

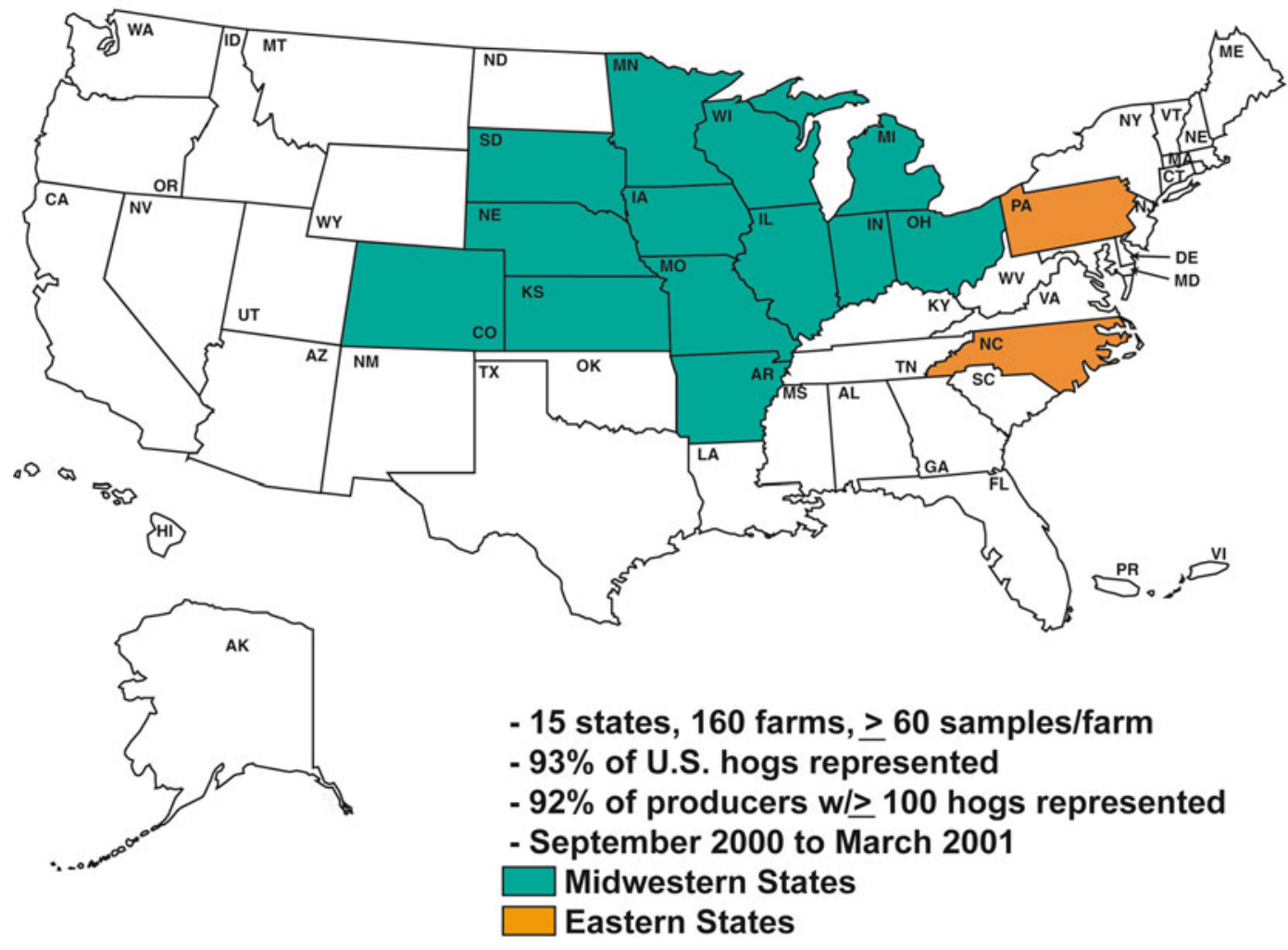

Fig. 14.2 States that participated in the swine study 
Ortiz Martinez et al. 2009). Thus, the isolation of pathogenic $Y$. enterocolitica from feces may be more difficult or less sensitive than other samples from swine or it may be that the prevalence is low. In 7 of the 15 participating states, at least one isolate was positive for the chromosomally encoded ail sequence, yielding an overall onfarm prevalence of $46.6 \%$. Chi-square analysis (Bhaduri et al. 2005) showed that four states, including Indiana ( $n=25$ [23.5\%] of 106), Iowa $(n=21$ [19.1\%] of 106), Minnesota $(n=18$ [16.8\%] of 106), and Nebraska $(n=29$ [27.3\%] of $106)$, had a significantly higher percentage (17$28 \%$ ) of ail-positive $Y$. enterocolitica than the number isolated (1-8\%) from Illinois $(n=4$ [3.7\%] of 106), Ohio ( $n=1$ [0.9\%] of 106), and South Dakota $(n=8[7.5 \%]$ of 106).

\subsubsection{Serotyping}

Serotyping is a valuable indicator of pathogenic potential, since both serotypes O:3 and O:5 harbor the ail gene (Carniel 2006). The ail-positive isolates were primarily serotype $0: 3$. The number and distribution of serotypes were statistically analyzed by the chi-square test (Bhaduri and Wesley 2006). Among seven states, 79 (74.2\%) of 106 of the isolates were serotype O:3, and $27(24.5 \%)$ of 106 of the isolates were serotype O:5. The O:3 serotype was exclusively distributed in Indiana $(n=24)$, Nebraska $(n=28)$, and South Dakota $(n=8)$. Serotype O:5 was found only in Illinois $(n=4)$ and Ohio $(n=1)$. Both serotypes were found in Iowa (O:3, $n=12$; $\mathrm{O}: 5, n=9)$ and Minnesota (O:3, $n=6$; O:5, $n=12)$. These results agree with previously published results but they differ from the observation that serotype 0:5 was predominant in swine (Bhaduri and Wesley 2006). Serotype O:3 strains are regarded as a major cause of human yersiniosis in the United States (CDC 2003). A significant finding of our study is that serogroup 0:3 (74.5\% of isolates) is the dominant virulence serogroup presently associated with swine in the United States.

\subsubsection{Presence of $p Y V$ in ail-Positive Y. enterocolitica}

Y. enterocolitica pathogenicity is dependent on chromosomal virulence genes, as well as virulence factors encoded by pYV (Carniel 2006). In the present study, the multiplex PCR assay targeting a key regulatory gene, $v i r F$, present on the pYV and the chromosomal ail gene showed that these ail-positive isolates (104) harbored pYV (YEP) and expressed pYV-associated phenotypes. The absence of pYV in two isolates demonstrated the instability of the pYV, which is easily lost on subculture and storage (Bhaduri 2001).

\subsection{4 pYV-Associated Virulence Characteristics}

One hundred and four $\mathrm{YEP}^{+}$isolates expressed pYV-encoded phenotypic characteristics, including colony morphology, crystal violet binding, low calcium response, Congo red uptake, autoagglutination, hydrophobicity, and YopE. These pYV-encoded virulence factors had been correlated with mouse pathogenicity and were used as direct markers for identifying pathogenic isolates of $Y$. enterocolitica among clinical and food sources (Bhaduri 2001).

\subsubsection{Genomic Analysis}

$X b a \mathrm{I}$ was the sole enzyme used in this study since it yields the most discriminating macrorestriction fragments for $Y$. enterocolitica. By PFGE, O:3 and O:5 ail-positive isolates could be distinguished. However, isolates were highly clonal within a serotype and exhibited minor variations that could not be correlated with geographic origin. Thus, isolates from different farms within the same state or from different states displayed nearly indistinguishable PFGE profiles. That O:3 and O:5 pulsotypes exhibit only minor variations within a serotype, regardless of geographic origin, indicates high clonality 
and that the genome of $Y$. enterocolitica is stable, an observation that concurs with others (Fredriksson-Ahomaa et al. 2011).

\subsubsection{Antibiotic Susceptibility of ail-Positive Isolates}

Antibiotic resistance of $106 \mathrm{YEP}^{+}$swine fecal isolates was studied to obtain baseline data of resistance patterns. A high degree of antibiotic susceptibility was observed in the sampled population of ail-positive $Y$. enterocolitica from swine feces. All of the strains ( $n=106)$ were susceptible to amikacin, amoxicillin, cefoxitin, ceftiofur, ceftriaxone, chloramphenicol, ciprofloxacin, gentamicin, kanamycin, nalidixic acid, streptomycin, and trimethoprim. Similar patterns of susceptibility were observed among strains isolated from pig tonsils in Switzerland, southern Germany, as well as in human strains (Bucher et al. 2008). Resistance to ampicillin was shown in all of the 106 isolates. Ampicillin resistance due to production of $\beta$-lactamases is well described in the literature (Bhaduri et al. 2009). Of the 106 isolates, $87.7 \%$ were resistant to cephalothin, and $27.4 \%$ were resistant to tetracycline. All of the isolates resistant to tetracycline were also resistant to cephalothin. Higher percentage of resistance to cephalothin (72-100\%) was found among four states; moderate resistance (13-69\%) to tetracycline was distributed among three states while no isolate from Nebraska was resistant. Likewise, Funk et al. (2000) in screening ail-bearing isolates of serotype 0:5 from hog tonsils in the Midwest concluded that the majority of isolates were resistant to ampicillin, penicillin, and cephalothin and could not correlate the presence of the ail gene with antimicrobial resistance. The presence or absence of the pYV did not have a significant effect on the resistance profile. These overall susceptibility/resistance results are consistent with what others have reported in the literature (Bucher et al. 2008; Funk et al. 2000).

\subsection{Conclusion}

Porcine isolates of $Y$. enterocolitica, which retained the chromosomal ail gene, pYV, and pYV-associated virulence phenotypic characteristics including cytotoxicity factor, YopE, were further analyzed to determine genotype antimicrobial profiles. Macrorestriction patterns demonstrated a high degree of clonality among isolates of the same serotype, regardless of geographic origin indicating stability of the genome. These pathogen isolates were sensitive to 13 of 16 antimicrobials. The results of this study support the hypothesis that swine are a significant potential reservoir for $Y$. enterocolitica strains. The varying presence of $Y$. enterocolitica from site to site suggests that management factors may influence on-farm prevalence of this organism. Modifications of such practices would ultimately reduce $Y$. enterocolitica transmission from pork products to humans. To fully understand the prevalence and risk factors associated with $Y$. enterocolitica infection in swine, additional investigations are needed of on-farm production, as well as postharvest processing systems.

Acknowledgments We thank Bryan Cottrell of the Molecular Characterization of Foodborne Pathogens Research Unit at the USDA, Eastern Regional Research Center, Wyndmoor, PA, and Ms. Laura Byl of the Preharvest Food Safety and Enteric Diseases Research Unit, USDA, National Animal Disease Center for technical assistance. The authors also thank Dr. John Phillips, Statistician, at the USDA/ARS/North Atlantic Area, who performed the statistical calculations and provided consultation on the statistical analyses.

\section{References}

Bhaduri S (2001) Pathogenic Yersinia enterocolitica. In: Labbe RH, Garcia-Alvarado JS (eds) Guide to foodborne pathogens. Wiley, New York

Bhaduri S, Wesley IV (2006) Isolation and characterization of Yersinia enterocolitica from swine feces recovered during the National Animal Health Monitoring System's Swine 2000 Study. J Food Prot 69: 2107-2112 
Bhaduri S, Wesley IV, Bush EJ (2005) Prevalence of pathogenic Yersinia enterocolitica strains in pigs in the United States. Appl Environ Microbiol 71: 7117-7121

Bhaduri S, Wesley IV, Richards H et al (2009) Clonality and antibiotic susceptibility of Yersinia enterocolitica isolated from US market weight hogs. Foodborne Pathog Dis 63:51-356

Bucher M, Meyer CB, Grotzbach B et al (2008) Epidemiological data on pathogenic Yersinia enterocolitica in southern Germany during 2000-2006. Foodborne Pathog Dis 5:273-280

Carniel E (2006) Y. enterocolitica and Y. pseudotuberculosis enteropathogenic yersiniae. In: Dworkin M, Falkow S, Rosenberg E, Stackebrandt E (eds) The prokaryotes, vol 6, chapter 3.3.13. Springer, New York

CDC (2006) Preliminary FoodNet data on the incidence of infection with pathogens transmitted commonly through food-10 states, United States, 2005. 55:392395. http://www.cdc.gov/mmwr/preview/mmwrhtml/ mm5514a2.htm. Accessed 1 Apr 2008

CDC (2011) Yersinia enterocolitica infections associated with pasteurized milk-Southwestern Pennsylvania, March-August 2011. MMWR 60:1428
CDC (2003) Yersinia enterocolitica gastroenteritis among infants exposed to chitterlings, Chicago, Illinois. MMWR 52:956-958

Fredriksson-Ahomaa M, Wacheck S, Bonke R et al (2011) Different enteropathogenic Yersinia strains found in wild boars and domestic pigs. Foodborne Pathog Dis 8:733-738

Funk JA, Troutt HF, Davis SA et al (2000) In vitro susceptibility of Yersinia enterocolitica isolated from the oral cavity of swine. J Food Prot 63:395-399

Laukkanen R, Ortiz Martinez P, Siekkinen K-M et al (2009) Contamination of carcasses with human pathogenic Yersinia enterocolitica 4/O:3 originates from pigs infected on farms. Foodborne Pathog Dis 6:681-688

Nesbakken T (2005) Yersinia enterocolitica. In: Fratamico PM, Bhunia AK, Smith JL (eds) Foodborne pathogens: microbiology and molecular biology. Caister Academic Press, Norfolk

Ortiz Martinez P, Fredriksson-Ahomaa M, Sokolova Y et al (2009) Prevalence of enteropathogenic Yersinia in Estonian, Latvian, and Russian (Leningrad region) pigs. Foodborne Pathog Dis 6:719-724

Scallen E, Hoekstra RM, Angulo FJ (2011) Foodborne illness acquired in the United States-major pathogens. Emerg Infect Dis 17:7-15 